\title{
Body composition and inflammation impact in non-small cell lung cancer patients treated by first-line immunotherapy
}

\author{
Cinzia Baldessari*,1 (D), Annarita Pecchi ${ }^{2}$, Raffaella Marcheselli ${ }^{3}$, Giorgia Guaitoli ${ }^{1}$, Riccardo \\ Bonacini ${ }^{2}$, Filippo Valoriani ${ }^{4}$, Pietro Torricelli ${ }^{2}$, Linda Reverberi ${ }^{4}$, Renata Menozzi ${ }^{4}$, Giuseppe \\ Pugliese ${ }^{1}$, Maria Giuseppa Vitale ${ }^{1}$, Roberto Sabbatini ${ }^{1}$, Federica Bertolini ${ }^{1}$, Fausto Barbieri ${ }^{1}$ \\ \& Massimo Dominici ${ }^{1}$ \\ ${ }^{1}$ Department of Oncology \& Hematology, Azienda Ospedaliero-Universitaria of Modena, Modena, 41124, Italy \\ ${ }^{2}$ Department of Radiology, University of Modena \& Reggio Emilia, Azienda Ospedaliero-Universitaria of Modena, Modena, 41124, \\ Italy \\ ${ }^{3}$ Department of Diagnostic, Clinical \& Public Health Medicine, University of Modena \& Reggio Emilia, Modena, Modena, 41124 , \\ Italy \\ ${ }^{4}$ Unit of Metabolic Disorder \& Clinical Nutrition, Department of Specialist Medicines, Azienda Ospedaliero-Universitaria of \\ Modena, Modena, 41124, Italy \\ *Author for correspondence: cinzia.baldessari@libero.it
}

Background: Immunotherapy changed the landscape of non-small cell lung cancer (NSCLC). Efforts were made to implement its action. This study aims to describe body composition, nutritional and inflammatory status in NSCLC patients treated by first-line immunotherapy, their correlation, variation and impact. Patients and methods: We retrospectively analyzed 44 consecutive patients who received pembrolizumab treatment. Results: During the therapy, inflammation and visceral fat increased, whereas muscle and subcutaneous fat decreased. Parameters related to inflammation had an interesting prognostic impact. High numbers of white blood cells remained significantly correlated with a high risk of death in multivariate model. Conclusion: For the best treatment choice, a combination of clinical and biological factors will be most likely be necessary. Prospective and larger studies with a multidimensional approach are needed.

Lay abstract: Inflammation and malnutrition in cancer patients may affect the immune system and response to therapy. We noticed an increase in inflammation and visceral fat and a decrease in muscle and subcutaneous fat during therapy. No variation showed a significant correlation with survival. Muscle mass, adipose tissue and body mass index do not confirm any prognostic impact or relationship with response to therapy. More interesting results were observed with parameters related to inflammation. Probably, for the best treatment choice, a combination of clinical and biological factors will be necessary. Further studies with a multidimensional approach are needed to propose the best treatment and the best support to everyone.

Tweetable abstract: Body composition, nutritional and inflammatory status changed during first-line immunotherapy on NSCLC patients. Inflammation has interesting prognostic implications. Combined with other factors, these clinical characteristics may be important to optimize the care of patients.

First draft submitted: 13 February 2021; Accepted for publication: 5 August 2021; Published online: 21 October 2021

Keywords: body composition • immunotherapy • inflammatory status • non-small cell lung cancer $\bullet$ nutritional status

Future Medicine 


\section{Graphical abstract:}

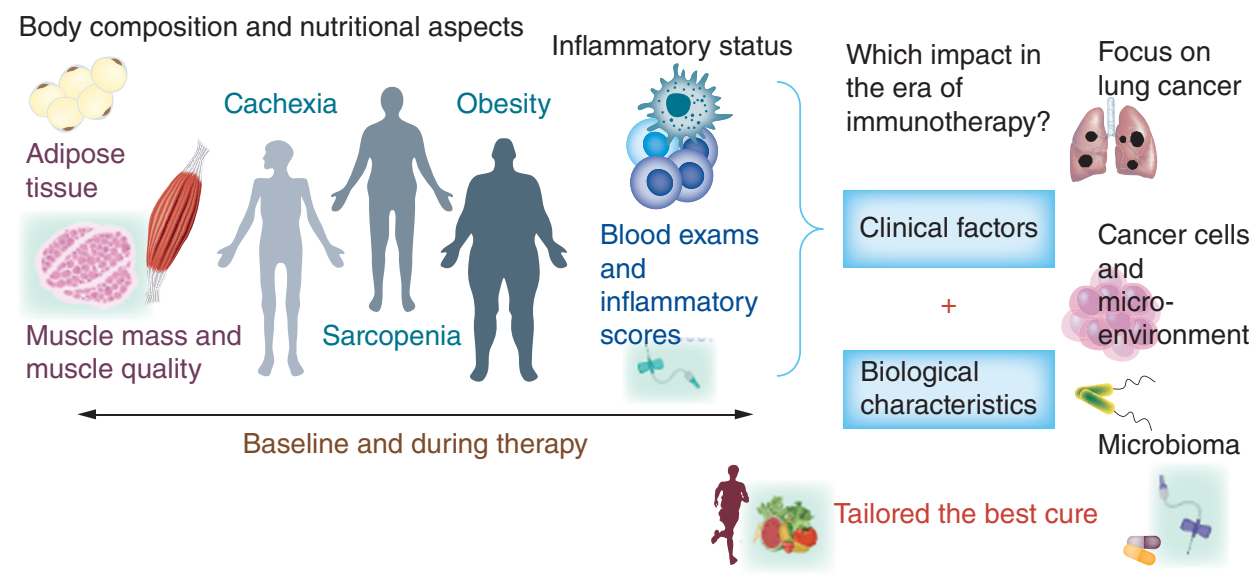

Lung cancer is the most important cause of cancer-related death worldwide and is frequently only diagnosed at advanced stages [1,2]. In recent years, immunotherapy (IT) has witnessed an important therapeutic change with the approval of nivolumab, pembrolizumab (for PD-L1 positive patients) and atezolizumab in pretreated patients [3-6] with non-small cell lung cancer (NSCLC). First-line monotherapy with pembrolizumab was approved in patients with high PD-L1 expression ( $\geq 50 \%$ ) according to Keynote 024 trial [7], while nivolumab did not obtain approval (Supplementary Table 1) [8], and atezolizumab was approved in May 2020 by the US FDA for patients with high PD-L1 expression and no EGFR or $A L K$ genomic tumor aberrations [9]. Recently, the combination of chemotherapy plus immunotherapy achieved good results both in pretreated patients and first-line setting [10-17]. Also, the combinations of nivolumab plus ipilimumab obtained OS benefit [17]. Despite this, until the end of 2019, only pembrolizumab monotherapy was approved and reimbursed in Italy as the first-line therapy for NSCLC patients without $E G F R$ and $A L K$ alterations and PD-L1 expression $\geq 50 \%$. Agenzia Italiana del Farmaco (AIFA) approved a combination of pembrolizumab with platinum and pemetrexed for patients with nonsquamous NSCLC with PD-L1 expression $<50 \%$ as front-line therapy in December 2019.

Several efforts were made to understand and implement the action of IT. When the host system fights against cancer, body composition and balance of nutritional and inflammatory status may be important for immune system's function: imbalance can lead to its malfunction [18-21]. Since the 1980s, unintentional weight loss up to the state of cachexia was related to worse prognosis in cancer patients undergoing chemotherapy [22]. In previous studies, depletion of muscle mass and increase of adipose tissue were described in the negative condition of 'sarcopenic obesity' [19,23]. Cancer sarcopenia and cachexia were sustained by inflammation in a 'vicious circle' that leads to an increase in chemotherapy toxicities [24-26]. Inflammatory markers, like CRP and neutrophil-to-lymphocyte ratio (NLR), were correlated to sarcopenia [27,28] and CRP was correlated to cancer cachexia [29].

It is of interest to focus on parameters describing sarcopenia, adipose tissue, nutritional and inflammatory status in relation with outcome in patients treated with IT. To achieve this aim, we selected a homogeneous subgroup of patients in first-line setting of therapy and took a picture of their current condition without the influence of other treatments. Patients with lung cancer who received first-line pembrolizumab appear very interesting to portray despite their complex conditions.

Some retrospective studies over the last few years, mostly by Japanese authors, have investigated this matter using different definitions of sarcopenia and concluded with divergent results (Table 1). All authors analyzed pretreated patients except Takada et al who included a small percentage of first-line patients (17/103) [30]. To our knowledge, this is the first study among western patients with lung cancer who underwent first-line IT describing their body composition and inflammatory status, their variation during IT and its relationship with outcome. 


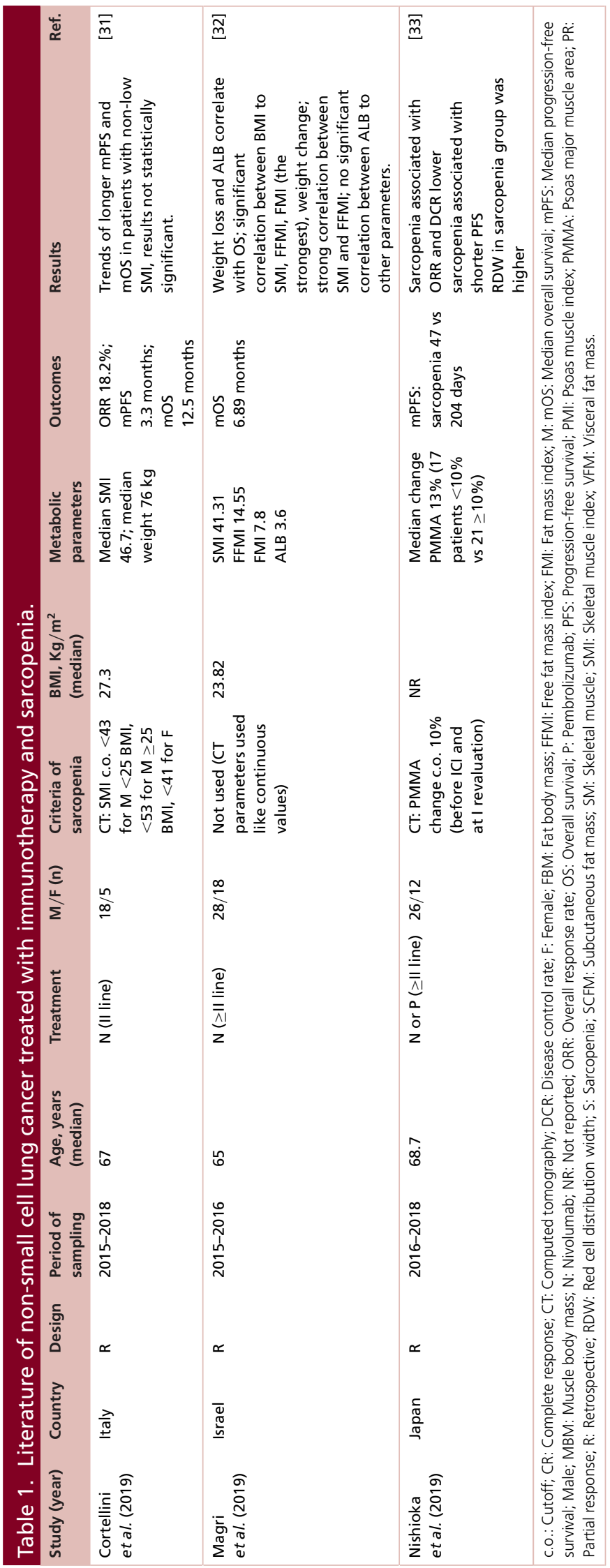




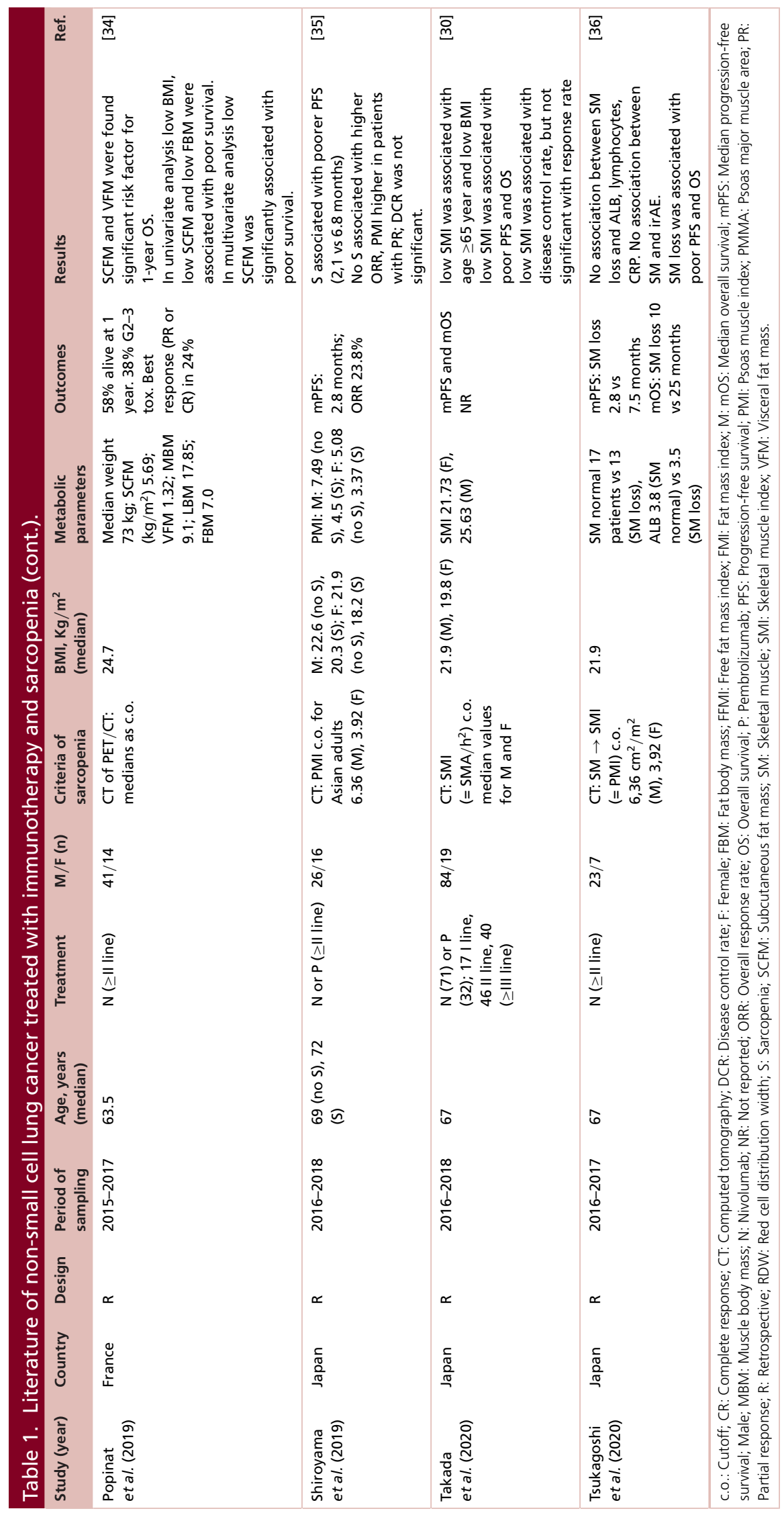


Figure 1. L3 axial CT scan images representative of the technique used. Using semi-automatic software, we can apply Hounsfield unit ranges to specifically select the muscle and the adipose components. With this method, we can calculate the area by manually tracing the region of interest that includes the PMA, SMA, SFA and VFA. PMA: Psoas muscle area; SFA: Subcutaneous fat area; SMA: Skeletal muscle area; VFA: Visceral fat area.

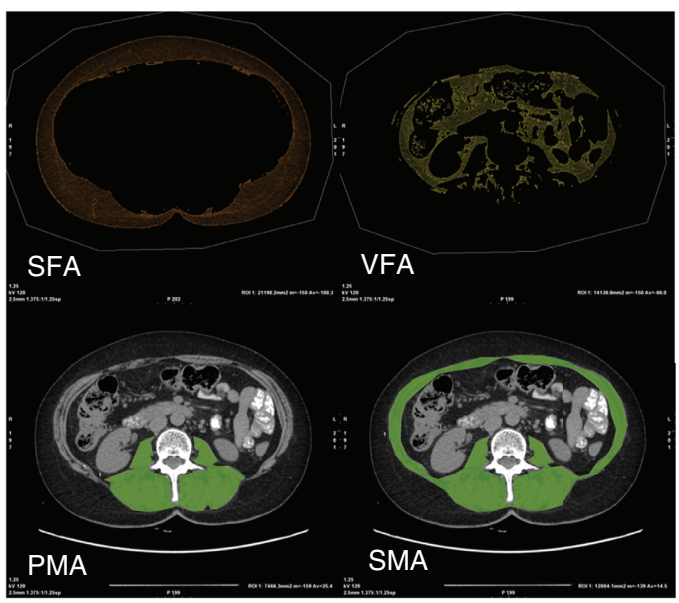

\section{Patients \& methods}

\section{Design of the study, inclusion criteria \& methods}

This retrospective and observational study involved patients consecutively treated with pembrolizumab as frontline therapy at Centro Oncologico Modenese between July 2017 and December 2018. Patients signed informed consent. Prior approval was obtained from the local ethic committee (prot. AOU 0019064/19, 03/07/2019) and authorization from the Azienda Ospedaliero-Universitaria of Modena (C.E. N. 403/2019). Clinical data were collected in a computerized database and anonymized before the analysis. Blood exams and computed tomography (CT) scan imaging were routinely made by patients according to clinical practice. Data cutoff was 24 February 2020. Patients were more than 18 years old, with histologically confirmed diagnosis of advanced NSCLC, received more than one infusion of pembrolizumab according to clinical practice and were not previously treated for advanced cancer. Available parameters considered for the analysis are reported in Table 2.

\section{Objective of the study}

The primary objective of the study was the description of the general characteristics of NSCLC patients treated by first-line IT, especially body composition, nutritional and inflammatory status at baseline and during IT. Then, we made a correlation between these parameters and between baseline parameters and outcome and response to IT.

\section{Technical aspects on CT scan imaging analysis}

Muscle mass and adipose tissue were measured on CT scan images routinely performed among patients before starting immunotherapy (basal CT scan) and at first restaging (obtained a median of 2.5 months after starting treatment, 95\% CI 2-3 months, standard deviation 0.99). Axial CT scan images were analyzed by a single operator using Advanced Workstation (AW) Server produced by General Electric (WI, USA) . In particular, the measures were taken at the level of a single slide at the height of the transverse process of L3. Muscle area of interest was selected in a semi-automatic manner using the range between -29 and +150 Hounsfield (HU) [58]. By positioning an elliptic region of interest (ROI) at the level of the right psoas muscle and the subcutaneous tissue of the ipsilateral lumbar region, the respective basal densities were assessed. Skeletal muscle index (SMI), as a measure of muscle mass, was obtained with skeletal muscle area (SMA) normalized for patients' height squared. Muscle attenuation (MA), as a measure of muscle quality, was the radio-density of the SMA measured in HU. Ratio between the psoas muscle density and the adipose subcutaneous tissue density permitted to calculate the intramuscular adipose tissue content (IMAC) of the psoas muscle, as another measure of muscle quality. Adipose tissue area was selected in a semi-automatic manner using the range between -180 and -30 . Tissue cross-sectional areas $\left(\mathrm{cm}^{2}\right)$ were calculated by the sum of pixel given multiplying by the pixel surface area. To calculate the index, the respective areas were normalized for patients' height squared. Total fat index (TFI) was obtained by the sum of visceral and subcutaneous fat areas (VFA and SFA, respectively) normalized for height squared, visceral fat index (VFI) and subcutaneous fat index (SFI). Visceral adiposity (VSR) was VFA/SFA ratio. Figure 1 is representative of the technique used and shows the selection of fat and muscle areas: SFA, VFA, psoas muscle area (PMA) and SMA. 
Table 2. Methods, parameters and values used in our analysis with relative references.

\begin{tabular}{|c|c|c|c|c|c|c|c|}
\hline \multirow{2}{*}{$\begin{array}{l}\text { Body } \\
\text { composition }\end{array}$} & \multirow[t]{2}{*}{ Methods } & \multirow[t]{2}{*}{ Parameters } & \multicolumn{2}{|c|}{ Timing therapy } & \multirow{2}{*}{$\begin{array}{l}\text { Cutoffs or normal } \\
\text { values }\end{array}$} & \multirow[t]{2}{*}{ Study (year) } & \multirow[t]{2}{*}{ Ref. } \\
\hline & & & pre & $\operatorname{in}^{\dagger}$ & & & \\
\hline \multirow[t]{5}{*}{ Muscle mass } & \multirow[t]{5}{*}{ CT-scan at L3 } & Psoas muscle index & $\mathrm{x}$ & $\mathrm{x}$ & M: 6.36; F: 3.92 & Shiroyama et al. (2019) & [35] \\
\hline & & $\begin{array}{l}\text { Total muscle } \\
\text { attenuation }\end{array}$ & $x$ & $x$ & $\mathrm{BMI} \geq 25: 33,<25: 41$ & Martin et al. (2013) & [37] \\
\hline & & $\operatorname{SMI}\left(\mathrm{cm}^{2} / \mathrm{m}^{2}\right)$ & $\mathrm{x}$ & $\mathrm{x}$ & M: 55; F: 39 & Fearon et al. (2011) & [38] \\
\hline & & $\begin{array}{l}\text { Free fat mass index } \\
\left(\mathrm{kg} / \mathrm{m}^{2}\right)\end{array}$ & $\mathrm{x}$ & $\mathrm{x}$ & M: 17; F: 15 & Cederholm et al. (2015) & [39] \\
\hline & & IMAC psoas & $x$ & & M: -0.358; F: -0.229 & Kobayashi et al. (2017) & [40] \\
\hline \multirow[t]{5}{*}{ Adipose tissue } & \multirow[t]{5}{*}{ CT-scan at L3 } & $\begin{array}{l}\text { Visceral fat area } \\
\left(\mathrm{kg} / \mathrm{cm}^{2}\right)\end{array}$ & $x$ & $\mathrm{x}$ & M: 163.8; F: 80.1 & Doyle et al. (2013) & [41] \\
\hline & & $\begin{array}{l}\text { Visceral fat index } \\
\left(\mathrm{cm}^{2} / \mathrm{m}^{2}\right)\end{array}$ & $x$ & $\mathrm{x}$ & M: 52.9; F: 51.5 & Ebadi et al. (2017) & [42] \\
\hline & & $\begin{array}{l}\text { Subcutaneous fat } \\
\text { index }\left(\mathrm{cm}^{2} / \mathrm{m}^{2}\right)\end{array}$ & $x$ & $\mathrm{x}$ & M: 50; F: 42 & Ebadi et al. (2017) & [42] \\
\hline & & $\begin{array}{l}\text { Total fat index } \\
\left(\mathrm{cm}^{2} / \mathrm{m}^{2}\right)\end{array}$ & $\mathrm{x}$ & $\mathrm{x}$ & M: 107.7; F: 102.2 & Ebadi et al. (2017) & [42] \\
\hline & & $\begin{array}{l}\text { Visceral adiposity } \\
\text { (visceral subcuta- } \\
\text { neous } \\
\text { ratio) }\end{array}$ & $x$ & $x$ & M: $1.325 ; F: 0.710$ & Kobayashi et al. (2017) & [40] \\
\hline \multicolumn{8}{|c|}{ Nutritional and inflammatory status } \\
\hline \multirow[t]{3}{*}{ Nutrition } & Clinical measure & Weight loss & $x^{\S}$ & $x^{\ddagger}$ & $5 \%$ and categories & $\begin{array}{l}\text { Fearon et al. (2011); Martin } \\
\text { et al. (2015) }\end{array}$ & {$[38,43]$} \\
\hline & Clinical measure & BMI & $\mathrm{x}$ & $\mathrm{x}$ & 25 and categories & $\begin{array}{l}\text { WHO (2011); Fearon } \\
\text { et al. 2011; Martin } \\
\text { et al. (2015) }\end{array}$ & {$[38,43,44]$} \\
\hline & Serum & ALB & $\mathrm{x}$ & $\mathrm{x}$ & n.v. $3.5-5.0$ & NA & \\
\hline \multirow[t]{5}{*}{ Inflammation } & Serum & $\begin{array}{l}\text { Red cell distribution } \\
\text { width }\end{array}$ & $\mathrm{x}$ & $\mathrm{x}$ & n.v. 12.6-15.8 & NA & \\
\hline & Serum & CRP & $\mathrm{x}$ & $\mathrm{x}$ & n.v. $0-0.7$ & NA & \\
\hline & Serum & WBC & $\mathrm{x}$ & $\mathrm{x}$ & n.v. $4000-10900$ & NA & \\
\hline & Serum (derived) & $\begin{array}{l}\text { Neutrophil-to- } \\
\text { lymphocyte } \\
\text { ratio }\end{array}$ & $x$ & $\mathrm{x}$ & 3.8 & Svaton et al. (2018) & [45] \\
\hline & Serum (derived) & $\begin{array}{l}\text { Platelet-to- } \\
\text { lymphocite } \\
\text { ratio }\end{array}$ & $\mathrm{x}$ & $\mathrm{x}$ & 169,1 & Svaton et al. (2018) & [45] \\
\hline \multicolumn{8}{|c|}{ Nutrition and inflammation prognostic scores } \\
\hline GPS & Serum (derived) & ALB, CRP & $\mathrm{x}$ & & Points $0-2$ & Forrest et al. (2003) & [46] \\
\hline mGPS & Serum (derived) & ALB, CRP & $x$ & & Points $0-2$ & $\begin{array}{l}\text { Proctor et al. (2011); Simmons } \\
\text { et al. (2015) }\end{array}$ & {$[47,48]$} \\
\hline CAR & Serum (derived) & ALB, CRP & $\mathrm{x}$ & & 0,2357 & Ni et al. (2018) & [49] \\
\hline ALI & $\begin{array}{l}\text { Serum (derived), } \\
\text { clinical measure }\end{array}$ & $\mathrm{BMI}, \mathrm{ALB}, \mathrm{CRP}$ & $\mathrm{x}$ & & 18 & $\begin{array}{l}\text { Ozyurek et al. (2018); } \\
\text { Shiroyama et al. (2018) }\end{array}$ & {$[50,51]$} \\
\hline mALI & $\begin{array}{l}\text { Serum (derived), CT } \\
\text { scan at L3 }\end{array}$ & SMI, ALB, CRP & $x$ & & 31.1 & Kim et al. (2016) & [52] \\
\hline $\mathrm{PNI}$ & Serum (derived) & ALB, L & $\mathrm{x}$ & & 45.5 & Shoji et al. (2019) & [53] \\
\hline PI & Serum (derived) & CRP, WBC & $\mathrm{x}$ & & Points $0-2$ & Kasymjanova et al. (2010) & [54] \\
\hline SII & Serum (derived) & $\begin{array}{l}\text { Platelet, } \\
\text { neutrophils, L }\end{array}$ & $\mathrm{x}$ & & 603.5 & Liu et al. (2019) & [55] \\
\hline CONUT & Serum (derived) & ALB, cholesterol, L & $\mathrm{x}$ & & 2 & Takamori et al. (2019) & [56] \\
\hline GNRI & $\begin{array}{l}\text { Serum (derived), } \\
\text { clinical measure }\end{array}$ & ALB, weight & $x$ & & 98 & Shoji et al. (2018) & [57] \\
\hline \multicolumn{8}{|c|}{$\begin{array}{l}\dagger \text { At first revaluation of the disease (median after } 2 \text { months from starting immunotherapy). } \\
\text { ¥Measurement at the last immunotherapy's infusion. } \\
\S_{\text {In the past } 6 \text { months. }} \\
\text { ALI: Advance lung cancer inflammation index; CAR: CRP and ALB ratio; CONUT: Controlling nutritional status; CT: Computed tomography; F: Female; GNRI: Preoperative geriatric } \\
\text { nutritional risk index; GPS: Glasgow Prognostic Score; IMAC psoas: Intra-muscle adipose tissue content of psoas; L: Lymphocytes; M: Male; mALI: Modified ALI; mGPS: Modified GPS; } \\
\text { NA: Not applicable; n.v.: Normal value; Pl: Prognostic index; PNI: Prognostic nutritional index; SIl: Systemic Immune-Inflammation Index; SMI: Skeletal muscle index; WBC: White blood } \\
\text { cell. }\end{array}$} \\
\hline
\end{tabular}




\section{Statistical analysis}

Descriptive statistics are presented as median, range and percentiles for continuous variables and values and percentage for categorical variables. Overall survival (OS) is defined as the time from the first immunotherapy infusion to the date of death or last follow-up. Patients alive at the last follow-up time were censored at the date of last contact. Progression-free survival (PFS) is defined as the time from the first immunotherapy infusion to the date of progression or last follow-up. Survival analysis was made with Kaplan-Meier method. Log-rank test was used on survival curves to compare difference between values over and under the selected literature cut-offs or normal values (Table 2). We calculated the receiver operating characteristic (ROC) curves of selected parameters searching the best cut-offs for our population. However, owing to the small cases series and the lack of statistical significance of the majority of ROC curves, we chose to rely on values already reported in the literature or normal laboratory values. Responses to therapy were defined according to the response evaluation criteria in solid tumors (RECIST) version 1.1. Overall response rate is defined like stable disease (SD) plus partial response (PR). Wilcoxon signed-rank test was used to create box plots and to give the median test to compare the chosen parameters with responses. Fisher's exact test was used to examine the differences between categorical variables. Spearman's test was used to examine the correlation between parameters. Univariate and multivariate analysis to determine mortality hazard ratios (HR) were conducted using Cox proportional hazard models to identify significant predictors of mortality. Results are reported as HRs and 95\% CI. Analysis was performed using STATA 11 and MedCalc Version 19.0.3.

\section{Results}

\section{Baseline characteristics of patients}

Baseline characteristics of the 44 patients involved in the study are detailed in Table 3. In addition, we evidenced that the large majority of patients $(82 \%)$ used three or more drugs daily as concomitant medications, in particular, $66 \%$ proton pomp inhibitors and $36 \%$ antibiotics in the months before or in the first months of therapy. During IT, $73 \%$ used steroids, of which $20 \%$ were for toxicities. About a third of patients (36\%) was submitted to radiotherapy during IT. As to body composition, CT images were available for 40 patients at baseline and for 34 patients at the first radiological evaluation (performed after a median of 90 days after IT start). With focus on muscle mass, at baseline, we noticed that different percentage of patients had low level of SMI using different cut-offs available in literature: $52.5 \%$ by Fearon et al. [38], $42.5 \%$ by Martin et al. [37], 55\% by Mourtzakis et al. [59] and 47.5\% by Prado et al. [23].

\section{Variation of patients' characteristics during IT}

Variations between CT scan values and blood exams at baseline and first-restaging are reported in Table 4. First restaging was considered the first CT scans made during IT, if available, and blood exams scheduled in the same period or earlier if patients did not undergo any imaging evaluation. We noticed an increase of parameters related to inflammation such as white blood cell (WBC) and red blood cell distribution width (RDW) and VSR and a decrease of parameters related to muscle mass (SMI), muscle quality (MA) and subcutaneous fat mass (SFI). Dividing the variation rate of these parameters with the time between the treatment start and the first restaging, no significant differences were observed, except for an increase in ALB (Supplementary Table 2). No variation of the studied parameters showed a significant correlation with OS, but a trend was frequently noted: worse OS with a decrease of more than $10 \%$ of SFI and an increase of more than $10 \%$ of CRP $(p=0.09$ and $p=0.06$, respectively; Supplementary Figure 1).

Among three selected parameters for muscle mass (psoas area, SMA and SMI) and adipose tissue (subcutaneous fat, visceral fat and VSR), none of the variation resulted significant in correlation with ORR and progressive disease (PD), except for the decrease of psoas muscle area among patients with PD compared to a slight increase in the median values of the same parameter in patients with SD or PR. Also, SMA and SMI showed a greater decrease among patients with PD like best response, with a trend toward significance ( $p=0.07$; Supplementary Figure 2).

\section{Correlation between different characteristics of patients}

Spearman's coefficients and significant p-value for correlation between different patients' characteristics are reported in Figure 2. Supplementary Table 3 reported all values calculated. Interesting correlations were those regarding parameters not included or combined in the formula for scores' calculation. For example, age was related with muscle quality (total MA), BMI with muscle mass (SMI), ALB with muscle quality (total MA) and inflammatory parameters (WBC, CRP, NLR and Systemic Immune-Inflammation Index [SII]) and CRP with inflammatory and 
Table 3. Baseline characteristics of patients regarding general characteristics, nutritional status, body composition, inflammatory status and nutritional and inflammatory scores.

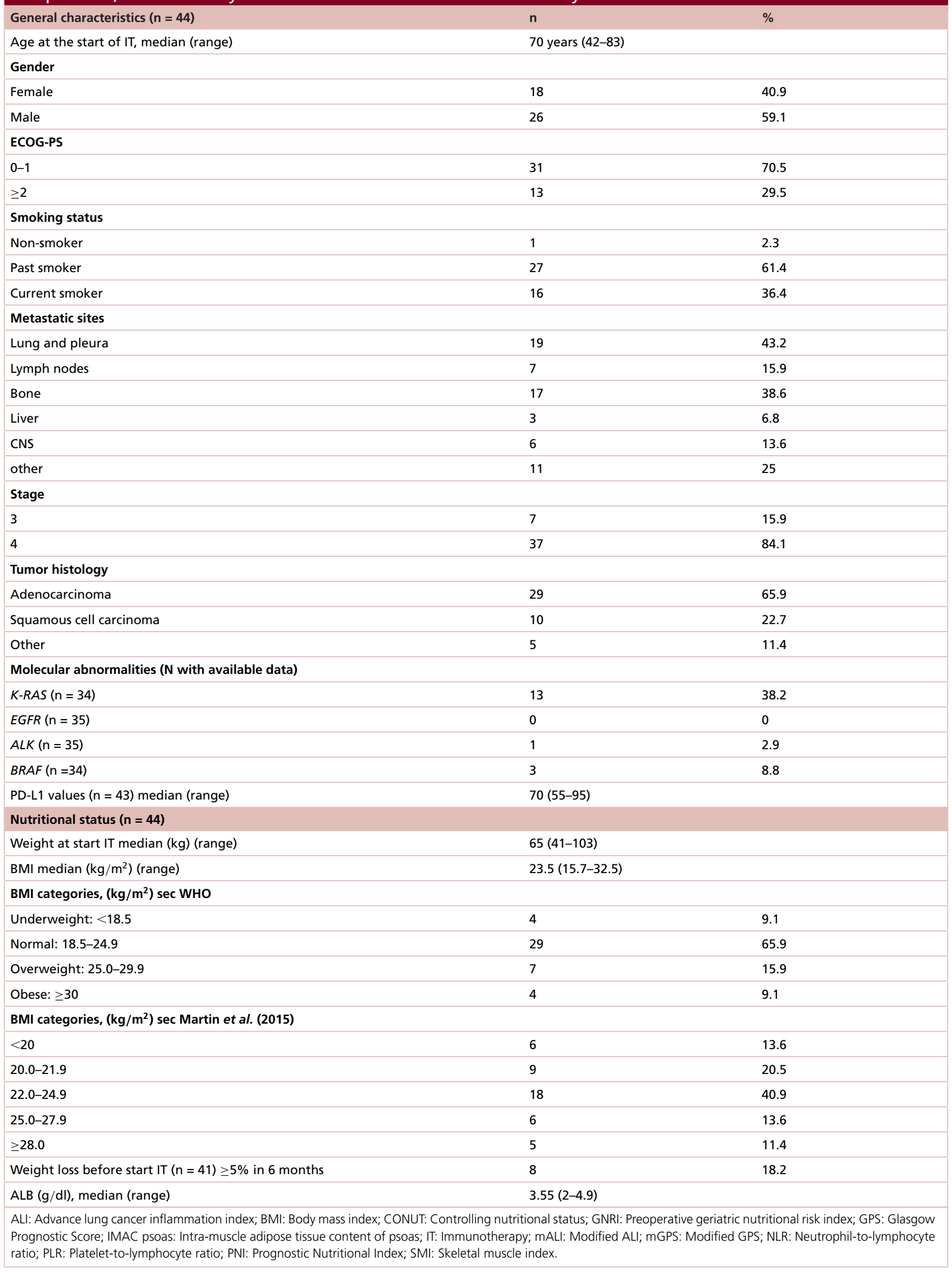


Table 3. Baseline characteristics of patients regarding general characteristics, nutritional status, body composition, inflammatory status and nutritional and inflammatory scores (cont.).

Nutritional status $(n=44)$

Basal Body composition characteristics $(n=40)$

Median (Range)

Muscle

Psoas area

$12(6.6-29.6)$

Psoas muscle index

$4.5(2.8-10)$

Region of interest psoas

$53.4(32.6-72.7)$

Total muscle attenuation

34 (13-51.6)

Total skeletal muscle area

$122.2(77.8-180.2)$

SMI - Total

$45.5(31.6-65.3)$

- Male $(n=23)$

- Female $(n=17)$

48.1 (36-65.3)

Free fat mass

$42.2(31.6-57.9)$

Free fat mass index

42.7 (29.4-60.1)

IMAC psoas

$15.9(11.6-22.2)$

Adipose tissue

$-0.5(-1-0.3)$

Visceral fat area

130.1 (13.9-476.7)

Visceral fat index

48.4 (5.2-139.3)

Subcutaneous fat area

135.6 (33.7-261.1)

Subcutaneous fat mass

47.1 (13.2-104.8)

Region of interest subcutaneous tissue

$-103.8(-126.1-64.2)$

Total fat index

$96.6(18.8-209.5)$

Fat mass

$22.6(13.2-39.2)$

Fat mass index

8 (4.8-13.8)

Visceral adiposity

$0.9(0.2-3.4)$

Inflammatory status (n data available)

Median (Range)

Blood exams

White blood cell $(/ \mathrm{mmc})(n=44)$

9425 (3980-43770)

Neutrophils $(/ \mathrm{mmc})(\mathrm{n}=44)$

7022.5 (1830-40250)

Red blood cell distribution width (cv\%) $(n=44)$

$14.3(12.5-21.3)$

CRP (mg/dl) ( $\mathrm{n}=37)$

$2.6(<0.2-23.3)$

Blood derived data

$\operatorname{NLR}(n=32)$

5 (1.2-18.4)

$\operatorname{PLR}(n=32)$

$179.1(97.1-864.2)$

Nutritional and Inflammatory scores (N data available)

Values or Median (Range)

Inflammation

GPS ( $n=37)$

mGPS ( $n=37)$

14 (0), 17 (1), 6 (2 points)

Prognostic Index $(n=44)$

CRP and ALB ratio $(n=37)$

27 (0), 4 (1), 6 (2 points)

Systemic Immune-Inflammation Index $(n=32)$

24 (0), 14 (1), 6 (2 points)

$0.1(0-0.9)$

ALI ( $n=32)$ 1366.3 (206.5-9947.1)

mALI $(n=28)$

16.6 (3.6-70.6)

Nutrition

PNI ( $n=32)$

$29.3(6.4-128.6)$

CONUT $(n=17)$

$42.1(27.8-60.3)$

GNRI $(n=44)$

$3(0-10)$

ALI: Advance lung cancer inflammation index; BMI: Body mass index; CONUT: Controlling nutritional status; GNRI: Preoperative geriatric nutritional risk index; GPS: Glasgow Prognostic Score; IMAC psoas: Intra-muscle adipose tissue content of psoas; IT: Immunotherapy; mALI: Modified ALI; mGPS: Modified GPS; NLR: Neutrophil-to-lymphocyte ratio; PLR: Platelet-to-lymphocyte ratio; PNI: Prognostic Nutritional Index; SMI: Skeletal muscle index. 
Table 4. Variation of blood exams and CT scan values from baseline to first restaging ${ }^{\dagger}$

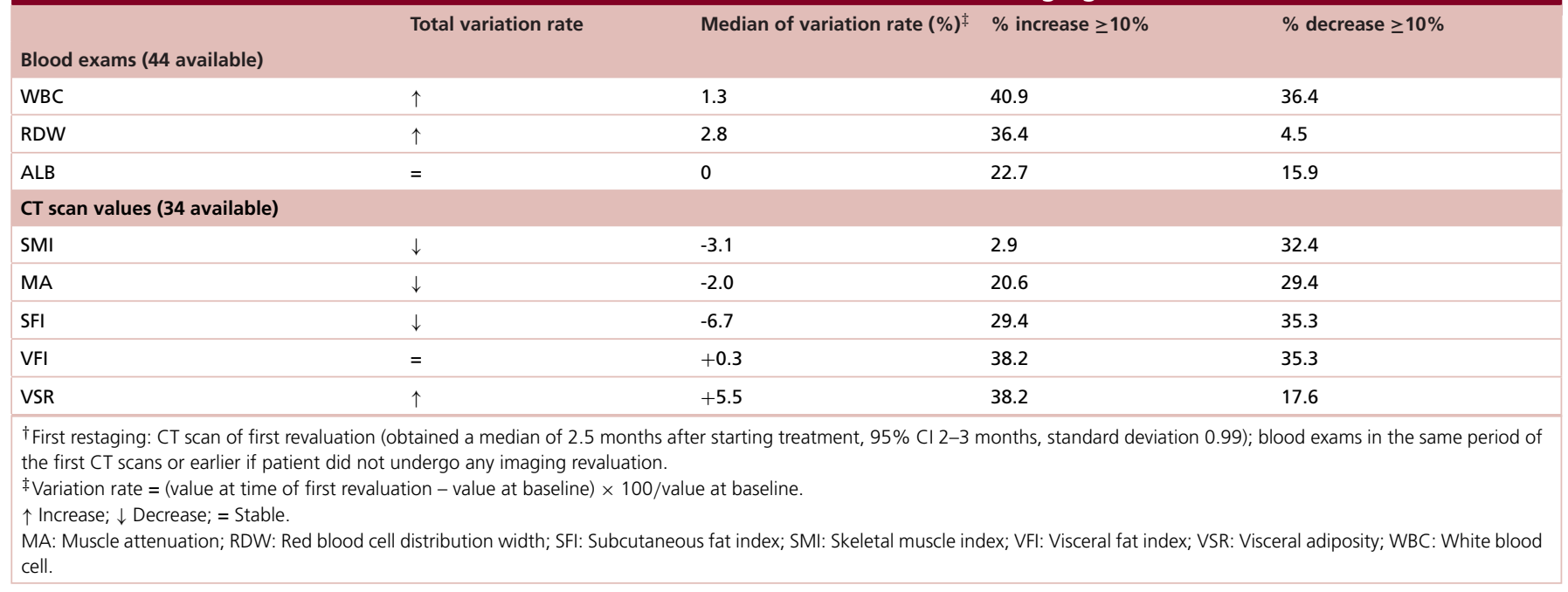

nutritional scores (SII, ALI, mALI, PNI and GNRI) and visceral adiposity (VSR) with inflammatory parameters (NLR, PLR, SII and ALI). In addition to the already cited correlation, muscle quality (total MA) was related with adipose tissue (SFI and VFI), muscle mass (SMI and psoas area) and PNI and muscle mass (SMI) with the nutritional score GNRI.

\section{Outcome with immunotherapy}

Median follow-up time was 265 days. Median overall survival (mOS) was 9.01 months (90\% CI 6.1-17.2 months; Figure 3A). Lack of patients selection may have influenced the result, but we observed a good percentage of patients in the 'tail' of the curve with a longer follow-up. One- and two-year OS rates were 45\% (95\% CI 33-60\%) and 40\% (95\% CI 24-54\%) respectively. Median progression free survival (mPFS) was 5.6 months (90\% 4.3-7.7 months; Figure 3B). One-year PFS was 41\% (95\% CI 26-55\%). There were five patients still on IT without PD and four stopped for toxicities or for physicians' or patient's decision without PD. Post-PD, one quarter of patients received chemotherapy, all with platinum doublet, the remaining started best supportive care.

Investigating general characteristics in relation with survival: sex (male vs female), age ( $<65$ vs $\geq 65$ years old and $<75$ vs $\geq 75$ years old), Eastern Cooperative Oncology Group (ECOG ; 0-1 vs $\geq 2$ ), presence of CNS metastasis (yes vs no, however there were only six patients with CNS metastasis in our analysis), weight loss (WL) ( $\geq 5 \%$ in 6 months vs $<5 \%$ ) and BMI ( $\leq 25$ vs $>25$ ) were not statistically significant correlated with survival in our patients series. Antibiotics use in the month before or in the first month of IT was associated with worse OS $(p=0.0012)$, whereas PPI use did not relate with it. None of the CT scan images parameter, neither about muscle mass nor adipose tissue, confirmed in our analysis a significant prognostic role. However, patients with a higher muscle mass (PMI, SMI, FFMI above the literature cut-offs) and a better muscle quality (higher muscle density, higher MA and IMAC) have a trend of better OS compared with others. Also, those with a higher SFI and TFI have a little trend of better OS. Regarding blood exams, a good prognostic value was confirmed for normal WBC, neutrophils, and ALB ( $\mathrm{p}=0.0217, \mathrm{p}=0.0121$ and $\mathrm{p}=0.0308$, respectively). No significance was found for NLR, PLR, CRP and RDW. Among nutrition and inflammation scores in our study, PI, ALI and mALI confirmed a significant prognostic role: low PI score (divided between 0 and $1-2$ points) was associated with better survival $(\mathrm{p}=0.0031)$ and low ALI $(<18)$ [50,51] and low mALI $(<31.1)$ [52] were associated with worse survival $(\mathrm{p}=0.0321$ and $\mathrm{p}=0.0446$, respectively). Moreover, ROC curves of selected parameters and related Kaplan-Meier OS curves with both values derived from ROC curves, medians and values derived from literature or normal laboratory values are reported in Supplementary Figures $3 \& 4$. We observed a similar trend among different Kaplan-Meier OS curves. This fact, though frequently without statistically significance, may reinforce the hypothesis that the results were due to the parameters rather than to the cut-offs used. However, the importance of the choice of the optimal cut-offs has to be underlined. We chose, mostly due to the small number of our case series, to rely on cut-offs already used for the main analysis. The use of medians may be another option, but statistical significance of the data were not always improved (as in Supplementary Figure 3). Univariate and Multivariate analysis on selected parameters calculated 

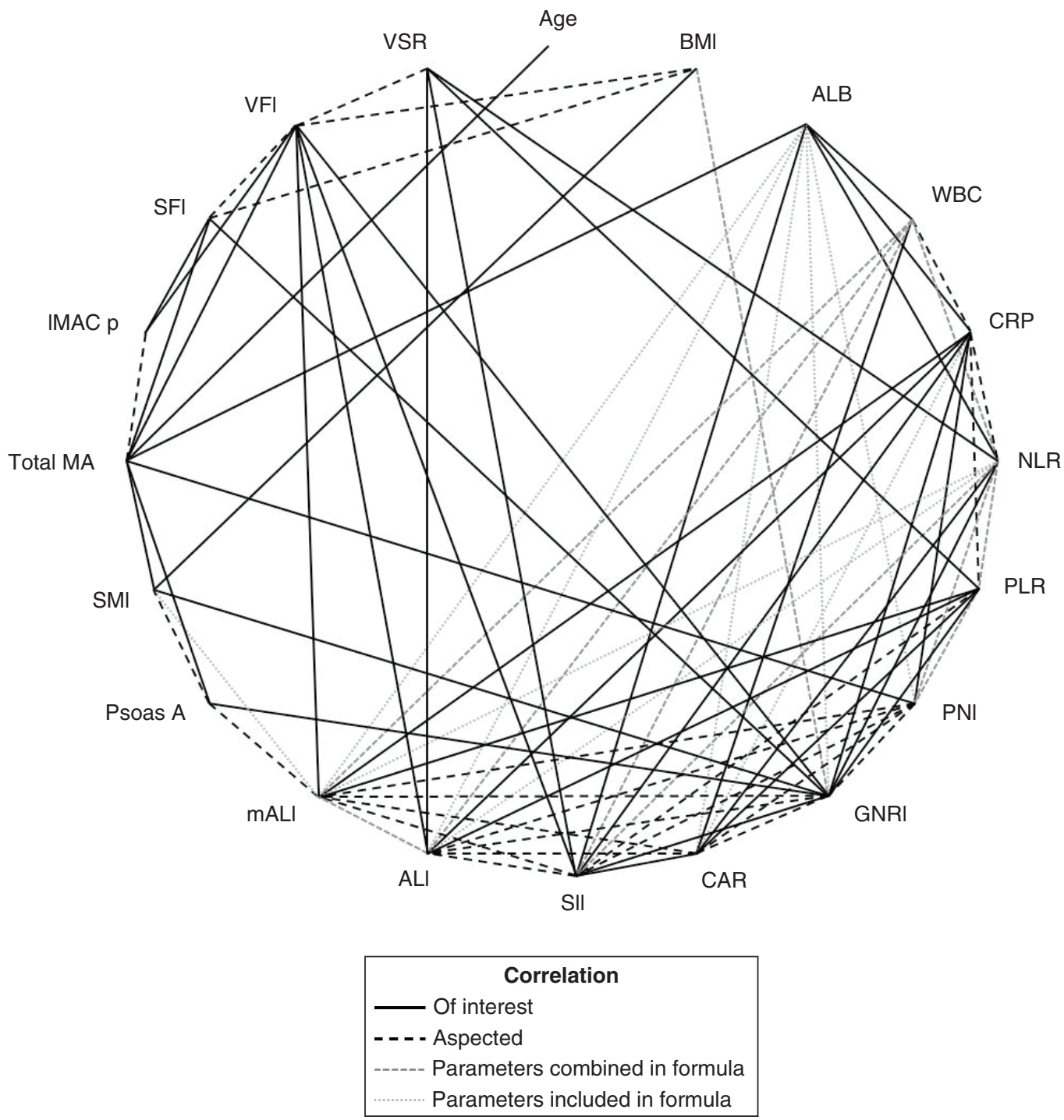

Figure 2. Correlation between different characteristics of patients.

ALI: Advance lung cancer inflammation index; CAR: CRP and ALB ratio; GNRI: Preoperative geriatric nutritional risk index; IMAC p: Intra-muscle adipose tissue content of psoas; MA: Muscle attenuation; mALI: Modified advance lung cancer inflammation index; NLR: Neutrophil-to-lymphocyte ratio; PLR: Platelet-to-lymphocyte ratio; PNI: Prognostic nutritional index; Psoas A: Psoas muscle area; SFI: Subcutaneous fat index; SII: Systemic Immune-Inflammation Index; SMI: Skeletal muscle index; VFI: Visceral fat index; VSR: Visceral adiposity; WBC: White blood cell.

with Cox proportional hazard model are reported in Table 5. In Univariate analysis ECOG, WBC, ALB, ALI and PI were significantly correlate with survival. In our multivariate model with the analysis of single parameters, without scores, high WBC remained significantly correlate with a high risk of death. Moreover, high BMI had a trend toward a low risk of death and higher VSR (like continuous variable) showed a trend toward a high risk of death. Considering WBC, ALB, CRP and NLR as continuous variables, the same evidence was confirmed in both univariate and multivariate analyses, though ALB lost significance in univariate analysis (Supplementary Table 4). All the results need to be considered with caution because of the reduced number of cases in the model.

ORR in our series was 56\% (0 complete response $+32 \%$ PR $+24 \%$ SD). Best response was PD in $44 \%$ of patients. No significant correlations were found between four baseline characteristics (two from CT scan imagines: SMI for investigate muscle mass and VSR for adipose tissue and two from blood exams: ALB for nutritional status and CRP for inflammatory status) and response (Supplementary Figure 5). 


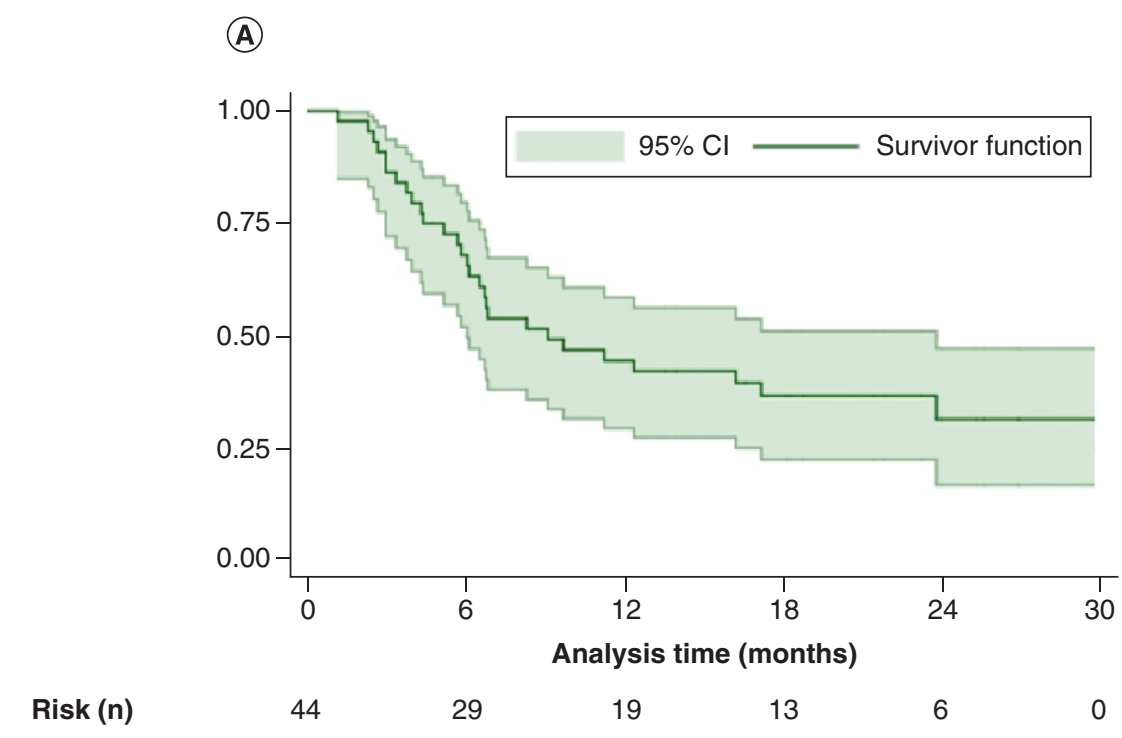

(B)

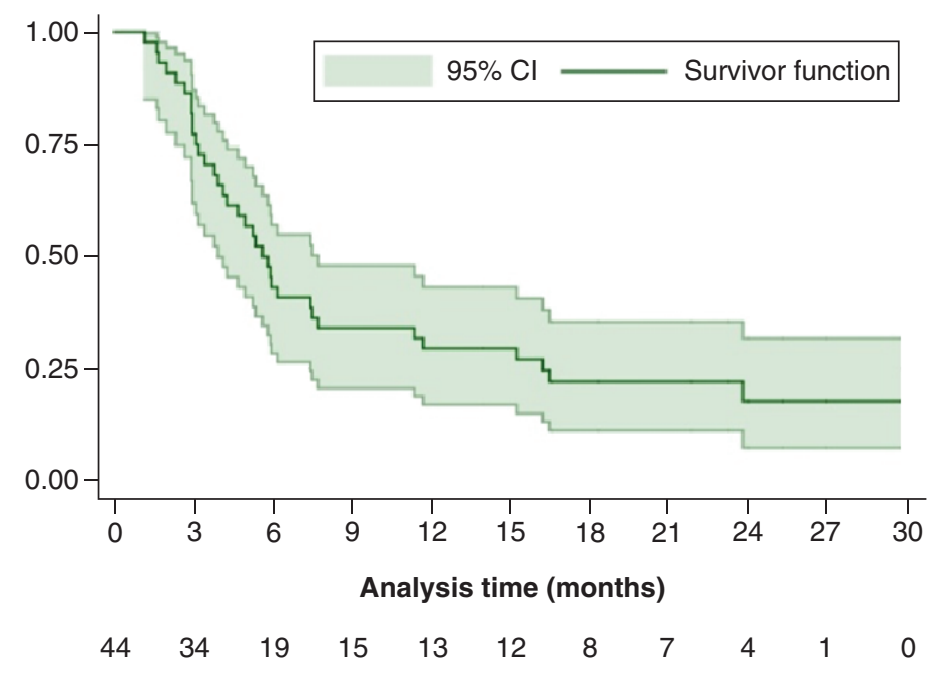

Figure 3. Overall Survival and progression-free survival in our patients. (A) Kaplan-Meier overall survival and (B) progression-free survival in our patients with $95 \% \mathrm{Cl}$ and number at risk under curves. Time in months.

\section{Discussion}

Inflammation has a prognostic role in some types of cancer and especially in those tumors where IT was first developed, like melanoma [60] and renal cell carcinoma [61]. It has to be underlined that the concept of inflammatory state used in our analysis has no relationship with the inflammatory state of the tumor and its microenvironment. Our analysis was based on clinical parameters without analyzing the biology of the tumor.

Our data confirmed that inflammatory status, investigated with different blood exams and scores, had prognostic significance in NSCLC patients treated by first-line IT. We could not refer specifically about its predictive value because our analysis lacks a comparison group, even if a predictive value may be supposed.

The PI score [54] based on CRP and WBC seemed superior in differentiating good from poor prognostic groups compared to the scores based on combination of circulating white cell count (NLR, PLR) or lymphocytes and ALB (PNI) [53]. These latter parameters did not reach statistical significance in our analysis. Scores combining CRP, ALB and body composition in term of BMI (ALI score) [50,51] and SMI (mALI) [52] performed well in giving prognostic information. CRP seemed to be a fundamental component to define prognosis on this group of 


\begin{tabular}{|c|c|c|c|c|}
\hline Characteristics & Variables & HR & $95 \% \mathrm{Cl}$ & p-value \\
\hline \multicolumn{5}{|c|}{ Univariate analysis } \\
\hline Sex & Female vs male & 0.78 & $0.37-1.67$ & 0.527 \\
\hline Smoking status & No or former vs yes & 0.72 & $0.33-1.56$ & 0.408 \\
\hline Age (years) & $\leq 65$ vs $>65$ & 0.81 & $0.37-1.80$ & 0.609 \\
\hline Met CNS & yes vs no & 2.40 & $0.90-6.39$ & 0.079 \\
\hline WBC & high vs normal & 2.34 & $1.11-4.96$ & 0.026 \\
\hline ALB & low vs normal & 2.25 & $1.05-4.80$ & 0.035 \\
\hline CRP & high vs normal & 2.36 & $0.81-6.90$ & 0.117 \\
\hline NLR & high vs normal & 1.57 & $0.67-3.62$ & 0.292 \\
\hline VSR & like continuous variable & 1 & $0.67-2.34$ & 0.511 \\
\hline ALI & $<$ c.o. vs $\geq$ c.o. (c.o. = 18) & 2.54 & $1.05-6.13$ & 0.038 \\
\hline mALI & $<$ c.o. vs $\geq$ c.o. $($ c.o. $=31.1)$ & 2.43 & $1-5-9.5$ & 0.051 \\
\hline $\mathrm{PI}$ & $1-2$ vs 0 points & 3.06 & $1.40-6.67$ & 0.005 \\
\hline $\mathrm{PNI}$ & $\leq$ c.o. vs $>$ c.o. (c.o. $=45.1)$ & 1.86 & $0.76-4.56$ & 0.173 \\
\hline \multicolumn{5}{|c|}{ Multivariate analysis } \\
\hline ECOG & & 1.76 & $0.69-4.56$ & 0.237 \\
\hline WBC & & 3.65 & $1.40-9.49$ & 0.008 \\
\hline ALB & & 1.38 & $0.59-3.21$ & 0.453 \\
\hline BMI & & 0.35 & $0.12-1.03$ & 0.056 \\
\hline
\end{tabular}

ALI: Advance lung cancer inflammation index; c.o.: Cutoff; ECOG: Eastern Cooperative Oncology Group; HR: Hazard ratio; mALl: Modified advance lung cancer inflammation index; PI: Prognostic index; PNI: Prognostic nutritional index; SMI: Skeletal muscle index; VSR: Visceral adiposity; WBC: White blood cell.

patients and normal values confirmed a better prognostic significance, as reported in the literature [62,63]. Normal WBC count and ALB values also had a positive prognostic significant. The recent retrospective study published by Matsubara et al., instead, found that PNI was an independent predictor of short time to treatment failure in NSCLC patients treated with atezolizumab and high NLR and high mGPS were independent prognostic factor for OS in this population [64].

Analysis of body composition both in muscle and in adipose tissue components, instead, did not confirm prognostic significance in our analysis. High values of parameters of muscle quantity (SMI) and quality (total MA) showed a trend of good prognostic significance, but without obtaining significant values. The same results on baseline skeletal muscle mass were recently published by Cortellini et al [31]. The major limitation of our analysis could be found in the low number of cases evaluated and the same limits was reported in the cited published study too [31]. In combination with other known important values, they reached the statistical significance (mALI). Also combined with other CT scan parameters, muscle mass has been reported to have a prognostic significance in patients treated by IT for different cancers, principally melanoma: a prognostic score based on CT scan analysis has been proposed. It includes tumor burden (1 point if $>90 \mathrm{~mm}$ ), SMI (1 point if $<53 \mathrm{~cm} / \mathrm{m}^{2}$ ) and nonpulmonary visceral metastasis ( 1 point if $>2$ ). The score predicts clinical benefit on anti-PD-1/PDL-1 therapy, independently from other conventional clinical-biological prognostic scores [65]. Another point of discussion is the different percentage of patients with low muscle mass that we found using the different cut-offs validated in the literature (excluding Asian cut-offs which are still different). The possibility of using different measures to assess sarcopenia, because of the non-univocal agreement, makes studies on the matter difficult to be compared. Adipose tissue parameters, on the other hand, appear to be distant from having a significant prognostic role, particularly if considered alone. There is little information in literature on the subject, except for the work by Ebadi et al [42]. 
Finally, considering the methods of body composition measurements, not dwelling on different techniques like bioelectrical impedance analysis (BIA) or dual energy x-ray absorptiometry (DXA), on CT or MRI, it has to be underlined that the limitations of a 2D measurement are known. For example, it has been shown that during weight loss, changes in visceral and subcutaneous adipose tissue are poorly assessed by a single slice (2D imaging) [66], while 3D imaging works well for fat [67]. Despite this, the 2D measurements techniques are still widely used in literature and in our center, we have the availability of this program.

BMI and weight loss deserve a special consideration. Probably, again owing to the small simple size, neither of them achieved statistical significance as prognostic factors in our study. Even if a trend could be described, we did not observe a significant 'obesity paradox', described instead in literature in different cancer types [68-70] including melanoma treated with immunotherapy and target therapy (but not with chemotherapy) [71]. Moreover, an Italian study involving different cancer types documented higher response rate and survival in overweight/obese patients [72]. However, when the patients were divided into various classes according to Martin et al. [43], BMI and weight loss showed significant value on overall survival in our analysis (Supplementary Figure 6). We cannot study the particular condition of 'sarcopenic obesity' specifically because we have only 11 patients with BMI $\geq 25$ of whom three with the criteria of sarcopenia and only four with BMI $\geq 30$ of whom one with 'sarcopenic obesity'. This phenomenon combines low muscle mass and high fat mass and was associated in literature with worst outcome [23] and more toxicity to IT [73].

Our data did not evidence a better outcome among female patients compared with male patients, showing only a possible trend, even if it was described in literature and reported in the meta-analysis by Conforti et al [74]. Neither ECOG PS reached statistical significance as prognostic factor, even it has known importance. Perhaps, an interesting analysis could be on the causes of an expired performance status: for burden/disease or for comorbidities. For the small number of cases from our series we could not draw conclusion based on our monocenter study, but we have participated to a large multicenter retrospective trial evaluating several clinic-pathological characteristics in relation with first-line pembrolizumab effectiveness that showed ECOG PS, bone and liver metastases as independent predictors of shortened OS [75].

The use of antibiotics in the near period before or at the start of IT had a negative prognostic role on survival in our analysis. The same evidence has been reported $[76,77]$ and underlined in a recent meta-analysis [78], demonstrating the scientific interest on the role of antibiotics in modifying the gut microbiome in relation with the efficacy of IT.

Our response rate (PR 32\%) is lower than that reported in the literature (Keynote 024 study [7] had objective response of $44.8 \%$ ), but we have consecutive as well as nonselected patients. Neither body composition baseline parameters (SMI for muscle mass and VSR for adipose tissue) nor blood baseline exams related to inflammatory status (ALB and CRP) correlated with response (ORR and PD).

Only the variation of muscle psoas area, between the investigated parameters, during first-line IT showed a significant association with response (divided between ORR and PD). SMA and SMI in the PD group showed a trend of greater decline (but not statistically significant).

The present results underline the importance of the systemic inflammatory response as a connecting factor between nutritional decline and poor outcome in cancer patients, as shown in previous studies that investigated the role of inflammatory scores [46,79]. Systemic inflammation may act as a 'common soil' promoting cancer progression [80] and its moderation may be important. In this scenario, systemic inflammation and body composition deterioration may not be faced by IT alone, but it will be probably necessary a multidrug and a multimodal approach to fight all aspects and to maximize treatments' effect. A detailed study on inflammation process in cancer is required to improve the efficacy of IT and to upgrade its function, which is established on the immune system and on a well-balanced host body.

From our data and literature analysis, we cannot draw definitive conclusions. However, we can speculate that inflammation has its role either alone and in combination with nutritional status and body composition for affecting the immune response against cancer and even more in the setting of IT. The negative role of body composition alterations on declining immunity has already been proposed [81]. Skeletal muscle interacts with immune response [82] and products IL-15, necessary for natural killer (NK) cell function [83]. These cells, expressing PD1, if activated in the tumor site, may be important in the action of anti PD1/PDL1 antibodies [84]. Adipose tissue has a known endocrine and inflammatory role for influencing tumor behavior [85]. An attempt to explain the double face of obesity, the increased risk of cancer and the apparently increased efficacy of PD1/PDL1 blockade, was made by Wang et al., on leptin signaling, T-cell aging with higher PD1 expression and dysfunction [86]. 


\section{Conclusion}

The vicious circle of chronic inflammation and malnutrition leading to sarcopenia and cachexia in cancer patients affects the immune system and response to therapy. IT, with its mechanism and complexity, has received significant attention because of the relevance of nutritional and inflammatory assessment for patients at baseline. Clinical parameters alone, however, do not include all aspects related to response to therapy and patients outcome. The lack of investigation of biological factors, such as PD-L1 and tumor mutational burden, in our analysis has to be underlined. We focus our attention on impact of body composition and inflammatory status (investigated with clinical parameters) in the setting of NSCLC patients treated by first-line IT. Probably, one universal and unique tool for the choice of the best treatment for every patient does not exist in this field, different from those cancers with a driver mutation or a driver molecular pathway to target. Important efforts were made by scientific communities to find various prognostic and predictive tools to use in treatment's choice. In the IT setting, either alone or in combination with other strategies, the best choice will be probably the combination of clinical and biological factors. To make a careful patients' screening before starting treatment and also an accurate patients' selection, incorporating early palliative care strategies to 'help' standard therapy should be the best strategy. Further clinical trials are required, possibly prospective and multicenter, to expand knowledge in the IT field combining clinical and biological factors each other and with preclinical and analytic studies. A step in the future IT will be the study of different lymphocytic subtypes and other cellular and noncellular components of the immune response against cancer and their dynamics during treatment. Two studies are ongoing in our center on this topic on melanoma and renal cell carcinoma. It will be interesting to investigate these aspects also in patients with different nutritional disorders, up to sarcopenia and cachexia, with the aim to research which components readily respond, which ones need to be stimulated to obtain an effective anti-tumor response, and which are not responded or exhausted in different patients' conditions. A multimodal approach based on nutritional, exercise and anti-inflammatory strategies should be subsequently studied to improve these aspects of the immune response against cancer to achieve maximum results in all patients and to propose the best treatment and/or the best support to everyone.

\section{Summary points}

- Inflammation and malnutrition in cancer patients may affect the immune system and response to therapy. The study of non-small cell lung cancer patients who have undergone first-line immunotherapy (IT) is interesting for these aspects.

- We noticed an increase in inflammation and visceral fat and a decrease in muscle mass, muscle quality and subcutaneous fat during therapy. No variation showed a significant correlation with survival.

- Muscle mass, adipose tissue and body mass index do not confirm a prognostic impact or relationship with response to therapy. More interesting results were observed with parameters related to inflammation.

- The use of antibiotics in the near period before or at the start of IT had a negative prognostic role on survival in our analysis.

- Neither body composition baseline parameters nor blood baseline exams related to inflammatory status correlated with response to IT, and only the variation of muscle psoas area during therapy showed a significant association with response.

- We can speculate that inflammation has its role either alone or in combination with nutritional status and body composition in affecting the immune response against cancer and even more in the setting of IT.

- Probably, for the best treatment choice, a combination of clinical and biological factors are necessary.

- Prospective and larger studies are needed with a multidimensional approach to propose the best cure and care to everyone.

Supplementary data

To view the supplementary data that accompany this paper please visit the journal website at: www.futuremedicine.com/doi/suppl/10.2217/imt-2021-0038

Author contributions

C Baldessari, F Bertolini, F Barbieri and A Pecchi conceived and designed the study. C Baldessari and R Marcheselli made statistical analysis. C Baldessari, G Guaitoli, R Bonacini, F Valoriani, L Reverberi and G Pugliese have collected data, revised the literature, wrote the manuscript and created tables and figures. P Torricelli, R Menozzi, MG Vitale, R Sabbatini and M Dominici supervised the study. 


\section{Acknowledgments}

The authors would like to thank all patients and their families.

Financial \& competing interests disclosure

The authors have no relevant affiliations or financial involvement with any organization or entity with a financial interest in or financial conflict with the subject matter or materials discussed in the manuscript. This includes employment, consultancies, honoraria, stock ownership or options, expert testimony, grants, or patents received or pending, or royalties.

No writing assistance was utilized in the production of this manuscript.

\section{Ethical conduct of research}

The study approval was obtained from the Local Ethic Committee (prot. AOU 0019064/19, date: 03/07/2019) and authorization from the 'Azienda Ospedaliero-Universitaria' of Modena (C.E. N. 403/2019). Patients gave written informed consent.

\section{References}

Papers of special note have been highlighted as: $\bullet$ of interest; $\bullet \bullet$ of considerable interest

1. Siegel RL, Miller KD, Jemal A. Cancer statistics, 2019. CA: Cancer J. Clin. 69(1), 7-34 (2019).

2. Goldstraw P, Chansky K, Crowley J et al. The IASLC Lung cancer staging project: proposals for revision of the TNM stage groupings in the forthcoming (eighth) edition of the TNM classification for lung cancer. J. Thorac. Oncol. 11(1), 39-51 (2016).

3. Brahmer J, Reckamp KL, Baas P et al. Nivolumab versus docetaxel in advanced squamous-cell non-small-cell lung cancer. N. Engl. J. Med. 373(2), 123-135 (2015).

4. Borghaei H, Paz-Ares L, Horn L et al. Nivolumab versus docetaxel in advanced nonsquamous non-small-cell lung cancer. $N$. Engl. J. Med. 373(17), 1627-1639 (2015).

5. Herbst RS, Baas P, Kim DW et al. Pembrolizumab versus docetaxel for previously treated, PD-L1-positive, advanced non-small-cell lung cancer (KEYNOTE-010): a randomised controlled trial. Lancet 387(10027), 1540-1550 (2016).

6. Rittmeyer A, Barlesi F, Waterkamp D et al. Atezolizumab versus docetaxel in patients with previously treated non-small-cell lung cancer (OAK): a phase 3, open-label, multicentre randomised controlled trial. Lancet 389(10066), 255-265 (2017).

7. Reck M, Rodríguez-Abreu D, Robinson AG et al. Pembrolizumab versus chemotherapy for PD-L1-positive non-small-cell lung cancer. N. Engl. J. Med. 375(19), 1823-1833 (2016).

-• This work brought immunotherapy in the first-line treatment of non-small cell lung cancer.

8. Carbone DP, Reck M, Paz-Ares L et al. First-line nivolumab in stage IV or recurrent non-small-cell lung cancer. N. Engl. J. Med. 376(25), 2415-2426 (2017).

9. Spigel D, de Marinis F, Giaccone G. IMpower1 10: interim OS analysis of a Phase III study of atezolizumab (atezo) vs platinum-based chemotherapy (chemo) as 1L treatment (tx) in PD-L1-selected NSCLC. Ann. Oncol. 30(5), v915 (2019).

10. Burtness B, Harrington KJ, Greil R et al. Pembrolizumab alone or with chemotherapy versus cetuximab with chemotherapy for recurrent or metastatic squamous cell carcinoma of the head and neck (KEYNOTE-048): a randomised, open-label, Phase 3 study. Lancet 394(10212), 1915-1928 (2019).

11. Gandhi L, Rodríguez-Abreu D, Gadgeel S et al. Pembrolizumab plus chemotherapy in metastatic non-small-cell lung cancer. N. Engl. J. Med. 378(22), 2078-2092 (2018).

12. Paz-Ares L, Luft A, Vicente D et al. Pembrolizumab plus chemotherapy for squamous non-small-cell lung cancer. N. Engl. J. Med. 379(21), 2040-2051 (2018).

13. West $\mathrm{H}, \mathrm{McCleod} \mathrm{M}$, Hussein $\mathrm{M}$ et al. Atezolizumab in combination with carboplatin plus nab-paclitaxel chemotherapy compared with chemotherapy alone as first-line treatment for metastatic non-squamous non-small-cell lung cancer (IMpower130): a multicentre, randomised, open-label, Phase 3 trial. Lancet Oncol. 20(7), 924-937 (2019).

14. Jotte R, Cappuzzo F, Vynnychenko I et al. Atezolizumab in combination with carboplatin and nab-paclitaxel in advanced squamous NSCLC (IMpower131): results from a randomized Phase III trial. J. Thorac. Oncol. 15(8), 1351-1360 (2020).

15. Papadimitrakopoulou V, Cobo M, Bordoni R et al. IMpower132: PFS and safety results with $1 \mathrm{~L}$ atezolizumab+ carboplatin/cisplatin + pemetrexed in stage IV non-squamous NSCLC. J. Thorac. Oncol. 13(Suppl. 10), S332-S333 (2018).

16. Socinski MA, Jotte RM, Cappuzzo F et al. Atezolizumab for first-line treatment of metastatic nonsquamous NSCLC. N. Engl. J. Med. 378(24), 2288-2301 (2018).

17. Hellmann MD, Paz-Ares L, Bernabe Caro R et al. Nivolumab plus ipilimumab in advanced non-small-cell lung cancer. N. Engl. J. Med. 381(21), 2020-2031 (2019).

18. Cehreli R, Yavuzsen T, Ates H, Akman T, Ellidokuz H, Oztop I. Can inflammatory and nutritional serum markers predict chemotherapy outcomes and survival in advanced stage nonsmall cell lung cancer patients? Biomed. Res. Int. 2019, 1648072 (2019). 
19. Baracos VE, Arribas L. Sarcopenic obesity: hidden muscle wasting and its impact for survival and complications of cancer therapy. Ann. Oncol. 29(Suppl. 2), ii1-ii9 (2018).

20. Calder PC. Feeding the immune system. Proc. Nutr. Soc. A72(3), 299-309 (2013).

21. De Rosa V, Di Rella F, Di Giacomo A, Matarese G. Regulatory T cells as suppressors of anti-tumor immunity: role of metabolism. Cytokine Growth Factor Rev. 35, 15-25 (2017).

22. Dewys WD, Begg C, Lavin PT et al. Prognostic effect of weight loss prior to chemotherapy in cancer patients. Eastern Cooperative Oncology Group. Am. J. Med. 69(4), 491-497 (1980).

23. Prado CM, Lieffers JR, McCargar LJ et al. Prevalence and clinical implications of sarcopenic obesity in patients with solid tumors of the respiratory and gastrointestinal tracts: a population-based study. Lancet Oncol. 9(7), 629-635 (2008).

24. Bozzetti F. Forcing the vicious circle: sarcopenia increases toxicity, decreases response to chemotherapy and worsens with chemotherapy. Ann. Oncol. 28(9), 2107-2118 (2017).

25. Hilmi M, Jouinot A, Burns R et al. Body composition and sarcopenia: the next-generation of personalized oncology and pharmacology? Pharmacol. Ther. 196, 135-159 (2019).

26. Argilés JM, Busquets S, Stemmler B, López-Soriano FJ. Cancer cachexia: understanding the molecular basis. Nat. Rev. Cancer 14(11), 754-762 (2014).

27. Bano G, Trevisan C, Carraro S et al. Inflammation and sarcopenia: a systematic review and meta-analysis. Maturitas 96, 10-15 (2017).

28. Öztürk ZA, Kul S, Türkbeyler İ, Sayıner ZA, Abiyev A. Is increased neutrophil lymphocyte ratio remarking the inflammation in sarcopenia? Exp. Gerontol. 110, 223-229 (2018).

29. Burney BO, Hayes TG, Smiechowska J et al. Low testosterone levels and increased inflammatory markers in patients with cancer and relationship with cachexia. J. Clin. Endocrinol. Metab. 97(5), E700-9 (2012).

30. Takada K, Yoneshima Y, Tanaka K et al. Clinical impact of skeletal muscle area in patients with non-small cell lung cancer treated with anti-PD-1 inhibitors. J. Cancer Res. Clin. Oncol. 146(5), 1217-1225 (2020).

31. Cortellini A, Verna L, Porzio G et al. Predictive value of skeletal muscle mass for immunotherapy with nivolumab in non-small cell lung cancer patients: a "hypothesis-generator" preliminary report. Thorac. Cancer. 10(2), 347-351 (2019).

- One of the first works and one of the rare works on the European population concerning the analysis of body composition, specifically analysis of muscle mass, in cancer patients treated with immunotherapy.

32. Magri V, Gottfried T, Di Segni M et al. Correlation of body composition by computerized tomography and metabolic parameters with survival of nivolumab-treated lung cancer patients. Cancer Manag. Res. 11, 8201-8207 (2019).

33. Nishioka N, Uchino J, Hirai S et al. Association of sarcopenia with and efficacy of anti-PD-1/PD-L1 therapy in non-small-cell lung cancer. J. Clin. Med. 8(4), 450 (2019).

34. Popinat G, Cousse S, Goldfarb L et al. Sub-cutaneous fat mass measured on multislice computed tomography of pretreatment PET/CT is a prognostic factor of stage IV non-small cell lung cancer treated by nivolumab. Oncoimmunology 8(5), e1580128 (2019).

- One of the rare work on European population and on the analysis of adipose tissue on cancer patient treated with immunotherapy.

35. Shiroyama T, Nagatomo I, Koyama $S$ et al. Impact of sarcopenia in patients with advanced non-small cell lung cancer treated with PD-1 inhibitors: A preliminary retrospective study. Sci. Rep. 9(1), 2447 (2019).

36. Tsukagoshi M, Yokobori T, Yajima $\mathrm{T}$ et al. Skeletal muscle mass predicts the outcome of nivolumab treatment for non-small cell lung cancer. Medicine (Baltimore) 99(7), e19059 (2020).

37. Martin L, Birdsell L, Macdonald $\mathrm{N}$ et al. Cancer cachexia in the age of obesity: skeletal muscle depletion is a powerful prognostic factor, independent of body mass index. J. Clin. Oncol. 31(12), 1539-1547 (2013).

38. Fearon K, Strasser F, Anker SD et al. Definition and classification of cancer cachexia: an international consensus. Lancet Oncol. 12(5), 489-495 (2011).

-• One of the most important works and an international consensus in the definition of cancer cachexia.

39. Cederholm T, Bosaeus I, Barazzoni R et al. Diagnostic criteria for malnutrition - an ESPEN consensus statement. Clin. Nutr. 34(3), 335-340 (2019).

40. Kobayashi A, Kaido T, Hamaguchi $\mathrm{Y}$ et al. Impact of visceral adiposity as well as sarcopenic factors on outcomes in patients undergoing liver resection for colorectal liver metastases. World J. Surg. 42(4), 1180-1191 (2018).

41. Doyle SL, Bennett AM, Donohoe CL et al. Establishing computed tomography-defined visceral fat area thresholds for use in obesity-related cancer research. Nutr. Res. 33(3), 171-179 (2013).

42. Ebadi M, Martin L, Ghosh S et al. Subcutaneous adiposity is an independent predictor of mortality in cancer patients. Br. J. Cancer 117(1), 148-155 (2017).

43. Martin L, Senesse P, Gioulbasanis I et al. Diagnostic criteria for the classification of cancer-associated weight loss. J. Clin. Oncol. 33(1), 90-99 (2015). 
44. World Health Organization Europe. Body mass index - BMI (2021). www.euro-who-int/en/health-topics/disease-prevention/nutrition/a-healthy-lifestyle/body-mass-index-bmi

45. Svaton M, Zemanova M, Skrickova J et al. Chronic inflammation as a potential predictive factor of nivolumab therapy in non-small cell lung cancer. Anticancer Res. 38(12), 6771-6782 (2018).

46. Forrest LM, McMillan DC, McArdle CS, Angerson WJ, Dunlop DJ. Evaluation of cumulative prognostic scores based on the systemic inflammatory response in patients with inoperable non-small-cell lung cancer. Br. J. Cancer 89(6), 1028-1030 (2003).

- This is one of the first studies on the analysis of prognostic scores related to inflammation in advanced cancer patients.

47. Proctor MJ, Morrison DS, Talwar D et al. An inflammation-based prognostic score (mGPS) predicts cancer survival independent of tumor site: a Glasgow inflammation outcome study. Br. J. Cancer 104(4), 726-734 (2011).

48. Simmons CP, Koinis F, Fallon MT et al. Prognosis in advanced lung cancer-a prospective study examining key clinicopathological factors. Lung Cancer 88(3), 304-309 (2015).

49. Ni XF, Wu J, Ji M et al. Effect of C-reactive protein/albumin ratio on prognosis in advanced non-small-cell lung cancer. Asia Pac. J. Clin. Oncol. 14(6), 402-409 (2018).

50. Ozyurek BA, Ozdemirel TS, Ozden SB et al. Does advanced lung inflammation index (ALI) have prognostic significance in metastatic non-small cell lung cancer? Clin. Respir. J. 12(6), 2013-2019 (2018).

51. Shiroyama T, Suzuki H, Tamiya M et al. Pretreatment advanced lung cancer inflammation index (ALI) for predicting early progression in nivolumab-treated patients with advanced non-small cell lung cancer. Cancer Med. 7(1), 13-20 (2018).

52. Kim EY, Kim N, Kim YS et al. Prognostic significance of modified advanced lung cancer inflammation index (ALI) in patients with small cell lung cancer comparison with original ALI. PLoS ONE 11(10), e0164056 (2016).

53. Shoji F, Takeoka H, Kozuma Y et al. Pretreatment prognostic nutritional index as a novel biomarker in non-small cell lung cancer patients treated with immune checkpoint inhibitors. Lung Cancer 136, 45-51 (2019).

54. Kasymjanova G, MacDonald N, Agulnik JS et al. The predictive value of pre-treatment inflammatory markers in advanced non-small-cell lung cancer. Curr. Oncol. 17(4), 52-58 (2010).

55. Liu J, Li S, Zhang S et al. Systemic immune-inflammation index, neutrophil-to-lymphocyte ratio, platelet-to-lymphocyte ratio can predict clinical outcomes in patients with metastatic non-small-cell lung cancer treated with nivolumab. J. Clin. Lab. Anal. 33(8), e22964 (2019).

56. Takamori $\mathrm{S}$, Toyokawa $\mathrm{G}$, Shimokawa $\mathrm{M}$ et al. A novel prognostic marker in patients with non-small cell lung cancer: musculo-immuno-nutritional score calculated by controlling nutritional status and creatine kinase. J. Thorac. Dis. 11(3), 927-935 (2019)

57. Shoji F, Miura N, Matsubara T et al. Prognostic significance of immune-nutritional parameters for surgically resected elderly lung cancer patients: a multicentre retrospective study. Interact. Cardiovasc. Thorac. Surg. 26(3), 389-394 (2018).

58. Mitsiopoulos N, Baumgartner RN, Heymsfield SB, Lyons W, Gallagher D, Ross R. Cadaver validation of skeletal muscle measurement by magnetic resonance imaging and computerized tomography. J. Appl. Physiol. 85(1), 115-122 (1998).

59. Mourtzakis M, Prado CM, Lieffers JR, Reiman T, McCargar LJ, Baracos VE. A practical and precise approach to quantification of body composition in cancer patients using computed tomography images acquired during routine care. Appl. Physiol. Nutr. Metab. 33(5), 997-1006 (2008)

60. Linee Guida AIOM 2018 MELANOMA (2018). www.aiom.it/wp-content/uploads/2018/11/2018_LG_AIOM_Melanoma.pdf

61. Motzer RJ, Mazumdar M, Bacik J, Russo P, Berg WJ, Metz EM. Effect of cytokine therapy on survival for patients with advanced renal cell carcinoma. J. Clin. Oncol. 18(9), 1928-1935 (2000).

62. Yang JR, Xu JY, Chen GC et al. Post-diagnostic C-reactive protein and albumin predict survival in Chinese patients with non-small cell lung cancer: a prospective cohort study. Sci. Rep. 9(1), 8143 (2019).

63. Iivanainen S, Ahvonen J, Knuuttila A, Tiainen S, Koivunen JP. Elevated CRP levels indicate poor progression-free and overall survival on cancer patients treated with PD-1 inhibitors. ESMO Open 4(4), e000531 (2019).

64. Matsubara T, Takamori S, Haratake $\mathrm{N}$ et al. The impact of immune-inflammation-nutritional parameters on the prognosis of non-small cell lung cancer patients treated with atezolizumab. J. Thorac. Dis. 12(4), 1520-1528 (2020).

65. Dercle L, Ammari $S$, Champiat $S$ et al. Rapid and objective CT scan prognostic scoring identifies metastatic patients with long-term clinical benefit on anti-PD-1/-L1 therapy. Eur. J. Cancer. 65, 33-42 (2016).

66. Shen W, Chen J, Gantz M, Velasquez G, Punyanitya M, Heymsfield SB. A single MRI slice does not accurately predict visceral and subcutaneous adipose tissue changes during weight loss. Obesity 20(12), 2458-2463 (2012).

67. Decazes P, Tonnelet D, Vera P, Gardin I. Anthropometer3D: automatic multi-slice segmentation software for the measurement of anthropometric parameters from CT of PET/CT. J. Digit. Imaging 32(2), 241-250 (2019).

68. Hakimi AA, Furberg H, Zabor EC et al. An epidemiologic and genomic investigation into the obesity paradox in renal cell carcinoma. $J$. Natl. Cancer Inst. 105(24), 1862-1870 (2013). 
69. Caan BJ, Meyerhardt JA, Kroenke $\mathrm{CH}$ et al. Explaining the obesity paradox: the association between body composition and colorectal cancer survival (C-SCANS study). Cancer Epidemiol. Biomarkers Prev. 26(7), 1008-1015 (2017).

70. Lam VK, Bentzen SM, Mohindra P et al. Obesity is associated with long-term improved survival in definitively treated locally advanced non-small cell lung cancer (NSCLC). Lung Cancer 104, 52-57 (2017).

71. McQuade JL, Daniel CR, Hess KR et al. Association of body-mass index and outcomes in patients with metastatic melanoma treated with targeted therapy, immunotherapy, or chemotherapy: a retrospective, multicohort analysis. Lancet Oncol. 19(3), 310-322 (2018).

72. Cortellini A, Bersanelli M, Buti $S$ et al. A multicenter study of body mass index in cancer patients treated with anti-PD-1/PD-L1 immune checkpoint inhibitors: when overweight becomes favorable. J. Immunother. Cancer. 7(1), 57 (2019).

73. Heidelberger V, Goldwasser F, Kramkimel N et al. Sarcopenic overweight is associated with early acute limiting toxicity of anti-PD1 checkpoint inhibitors in melanoma patients. Invest. New Drugs. 35(4), 436-441 (2017).

74. Conforti F, Pala L, Bagnardi V et al. Cancer immunotherapy efficacy and patients' sex: a systematic review and meta-analysis. Lancet Oncol. 19(6), 737-746 (2018).

75. Cortellini A, Tiseo M, Banna GL et al. Clinicopathologic correlates of first-line pembrolizumab effectiveness in patients with advanced NSCLC and a PD-L1 expression of $\geq 50 \%$. Cancer Immunol. Immunother. 69(11), 2209-2221 (2020).

76. Derosa L, Hellmann MD, Spaziano $\mathrm{M}$ et al. Negative association of antibiotics on clinical activity of immune checkpoint inhibitors in patients with advanced renal cell and non-small-cell lung cancer. Ann. Oncol. 29(6), 1437-1444 (2018).

77. Schett A, Rothschild SI, Curioni-Fontecedro A et al. Predictive impact of antibiotics in patients with advanced non small-cell lung cancer receiving immune checkpoint inhibitors: antibiotics immune checkpoint inhibitors in advanced NSCLC. Cancer Chemother. Pharmacol. 85(1), 121-131 (2020)

78. Huang XZ, Gao P, Song YX et al. Antibiotic use and the efficacy of immune checkpoint inhibitors in cancer patients: a pooled analysis of 2740 cancer patients. Oncoimmunology 8(12), e1665973 (2019).

79. McMillan DC. An inflammation-based prognostic score and its role in the nutrition-based management of patients with cancer. Proc. Nutr. Soc. 67(3), 257-262 (2008).

- Study on the importance and correlation of the combined management of nutritional and inflammation-related aspects.

80. Fidler IJ, Poste G. The "seed and soil" hypothesis revisited. Lancet Oncol. 9(8), 808 (2008).

81. Lutz CT, Quinn LS. Sarcopenia, obesity, and natural killer cell immune senescence in aging: altered cytokine levels as a common mechanism. Aging 4(8), 535-546 (2012).

82. Afzali AM, Müntefering T, Wiendl H, Meuth SG, Ruck T. Skeletal muscle cells actively shape (auto)immune responses. Autoimmun. Rev. 17(5), 518-529 (2018).

83. Quinn LS. Interleukin-15: a muscle-derived cytokine regulating fat-to-lean body composition. J. Anim. Sci. 86(Suppl. 14), E75-E83 (2008).

84. Pesce S, Greppi M, Tabellini G et al. Identification of a subset of human natural killer cells expressing high levels of programmed death 1 : a phenotypic and functional characterization. J. Allergy Clin. Immunol. 139(1), 335-346.e3 (2017).

85. Deng T, Lyon CJ, Bergin S, Caligiuri MA, Hsueh WA. Obesity, inflammation, and cancer. Annu. Rev. Pathol. 11, $421-449$ (2016).

- This review summarises several relationships between obesity, inflammation and cancer.

86. Wang Z, Aguilar EG, Luna JI et al. Paradoxical effects of obesity on T cell function during tumor progression and PD-1 checkpoint blockade. Nat. Med. 25(1), 141-151 (2019). 